\title{
Selección de conchas por cangrejos ermitaños (Decápoda: Anomura) en dos localidades del Pacífico Panameño
}

2.Ministerio de Educación, CEBG Ernestina Sucre Falcón. Maestría en Ecología de zonas costeras con orientación al manejo de los recursos costeros-marinos. cedenoc19@gmail.com https://orcid.org/0000-0002-1634-2342

\section{Págs.: 1 - 11}

Recibido: 6 /8/2020

Aprobado: 20/8/2020

\section{Resumen}

Durante septiembre 2014, se analizaron las preferencias (in situ) de tipos de conchas, en ejemplares de cangrejos ermitaños en Punta Pajarito, Área de Recursos Manejados Humedal Golfo de Montijo (Veraguas) y playa El Retén, Bahía de Parita (Herrera), en el Pacífico panameño. Se reporta que los cangrejos ermitaños tienen mayor preferencia por las conchas de la familia Naticidae, según datos recopilados es la concha más abundante en el área. En playa el Retén a 28 individuos de la familia Diogenidae, se les midieron parámetros como peso del individuo, peso y volumen de la concha. Al aplicar el coeficiente de correlación de Spearman a las variables peso del individuo y la relación peso- volumen de la concha, no se evidencia correlación entre el peso del organismo y la relación peso-volumen de la concha $(\mathrm{p}=0,386)$, por lo que la disponibilidad de conchas es el parámetro que mejor explica la ocupación de conchas en playa El Retén. Esta investigación constituye un primer esfuerzo en el área y permite un conocimiento base de los patrones ecológicos que interactúan en la selección de conchas por cangrejos ermitaños. 
Palabras claves: selección de conchas, cangrejos ermitaños, Naticidae, Diogenidae.

\begin{abstract}
During September 2014, preferences (in situ) shell type specimens, of hermit crabs in Punta Pajarito, Managed Resources Wetland Area Gulf of Montijo (Veraguas) and El Retén beach, Parita bay (Herrera) panamanian Pacific were analyzed. It is reported that hermit crabs have a greater preference for the shells of Naticidae family, possibly because this is the most abundant in the area shell. 28 individuals from the Diogenidae family were collected at the beach of El Retén, parameters such as individual weight, weight and volume of the shell were taken. When applying the Spearman correlation coefficient to the variables individual weight and weight-volume ratio of the shell, there is no evidence of a correlation between the weight of the organism and the weightvolume relationship of the shell ( $\mathrm{p}=0.386$ ), so the availability of shells is the parameter that best explains the occupation of shells at El Retén beach. This research constitutes a first effort in the area and allows a basic knowledge of the ecological patterns that interact in the selection of shells by hermit crabs
\end{abstract}

Keywords: selection of shells, hermit crabs, Naticidae, Diogenidae

\title{
Introducción
}

Los cangrejos ermitaños pertenecen a un grupo de crustáceos denominados Anomura, los cuales se caracterizan por tener el abdomen imperfectamente o incompletamente replegado por debajo del cefalotórax y a menudo reducido o asimétrico con atrofia de los pleópodos, pero siempre con urópodos (Fischer et al., 1995). Estos organismos representan un grupo representativo de la fauna marina, y son importantes en el mantenimiento del equilibrio dinámico, en los ecosistemas por sus interacciones ecológicas y también por el impacto en la estabilidad de las redes tróficas (Martínez, Campos y Bermúdez, 2012).

Debido a su blando abdomen y para una mayor protección en general, los cangrejos ermitaños han de buscar conchas vacías de gasterópodos para protegerse de sus enemigos y factores ambientales (Romero, 1973; Bertness, 1980; Cipresso, Zancaner y Jacobucci, 2009; Ribeiro et al., 2018). No obstante, en determinado momento de su vida, la concha se convierte en un factor limitante para el crecimiento y capacidad 
reproductiva del cangrejo, obligándolo a buscar una nueva concha que se ajuste mejor sus necesidades (Hazlett, 1996, Angel, 2000; Dahlet, Checon, Yokoyama y Turra, 2019).

Por lo tanto, la disponibilidad de conchas vacías de gasterópodos, frecuentemente es un recurso limitado y limitante para el crecimiento poblacional en cangrejos ermitaños (Guillén y Osorno, 1993; Murillo, Burbano y Olano, 2003; Pogio, 2007; Carranza, Segura, López y Rubio, 2008). Por consiguiente, debido a que la disponibilidad de conchas relacionada a la abundancia relativa de gasterópodos, su tasa de mortalidad y al estado de las mismas luego de su muerte (Bertness 1980), los cangrejos ermitaños pueden constituirse en un indicador indirecto de sobreexplotación de gasterópodos de interés gastronómico y comercial.

Referente a los criterios utilizados por los cangrejos ermitaños para la selección de conchas, diversos autores sostienen que la disponibilidad de conchas en una determinada área es el principal criterio de selección. (Monteforte y Leija- Tristán, 1990; Rendón- Salinas, 2000; Mantelatto y Domiciano, 2002; Arrasate- López, 2008; Sardar, Ghosh, Kumar, Bhattacharjee y Pal, 2019). Otros factores como la baja relación peso-volumen de la concha, con conchas de mayor volumen interno y más livianas, que ofrecen menos protección, pero menor gasto energético de transporte (Romero, 1973; Conover, 1978; Osorno, Fernández- Casillas y Rodríguez- Juárez, 1998; RendónSalinas, 2000; Angel, 2000; Mantelatto, Biagi, Meireles y Scelzo, 2007; ArrasateLópez, 2008; Argüelles- Tico, 2010), el tamaño de la concha (Murillo et al., 2003; Jeremy y Patria, 2020), la relación tamaño-peso de la concha (Biagi, Meireles, Scelzo y Mantelatto, 2006), así como el tamaño/peso del cangrejo ermitaño huésped (Biagi et al., 2006; Murillo et al., 2003), también se relacionan al proceso de selección de conchas.

Por lo tanto, debido a que los criterios utilizados por cangrejos ermitaños en la selección de conchas son desconocidos en la región, el propósito de la presente investigación fue correlacionar el peso de los cangrejos ermitaños en relación al peso/volumen de su concha, identificando in situ las conchas de mayor preferencia. De este modo, los datos obtenidos permiten establecer una línea base de conocimiento sobre los patrones que interactúan en la selección de conchas por cangrejos ermitaños, así como las conchas de mayor preferencia in situ. 


\section{Materiales y Métodos}

La presente investigación se realizó en dos localidades del Pacifico panameño: Punta Pajarito (7 42' 30.002" N y 81 11'40.178" W UTM), Área de Recursos Manejados Humedal Golfo de Montijo, provincia de Veraguas y playa El Retén ( $8^{\circ} 1^{\prime}$ 18.552" N y $80^{\circ} 26^{\prime} 12.174 "$ W, UTM), Bahía de Parita, provincia de Herrera. Playa El Retén se encuentra localizada en el corregimiento de Monagrillo, provincia de Herrera y caracteriza por la presencia de salineras, pesca artesanal (camarones, peces y conchas, principalmente) y el tradicional concurso anual de panderos y cometas, que se desarrolla entre los meses de enero a febrero.

Los cangrejos ermitaños se colectaron durante una gira a cada sitio en septiembre 2014, registrándose preferencia de concha, in situ. Para el muestreo se estableció una parcela de 100 metros lineales al borde de la zona intermareal, colectándose durante la marea baja los individuos sin ningún criterio de selección. Las conchas utilizadas por los cangrejos ermitaños se clasificaron hasta familia utilizando la guía de Keen (1971).

En playa El Retén, se seleccionó al azar, 31 individuos (no ovígeros) y sus conchas, a los cuales se le determinó peso del individuo y su concha con una balanza electrónica de $\pm 0.1 \mathrm{~g}$, de precisión y el volumen interno de la concha por desplazamiento volumétrico de agua utilizando una probeta de $100 \mathrm{ml}$, utilizando el procedimiento propuesto de Guillén y Osorno (1993). Para extraer a los cangrejos ermitaños de su concha se siguieron dos procedimientos: para individuos grandes que no se ocultaban totalmente en las mismas, se tomaban firmemente de quelas y el resto de la parte anterior del cuerpo y se extraían suavemente con una pinza. Para individuos que se ocultaban totalmente en sus conchas, fueron retirados aplicándole calor al ápice del caparazón. Los individuos colectados y sus conchas se clasificaron hasta familia siguiendo la guía de Fischer et al. (1995) y Keen (1971), respectivamente, y los datos obtenidos se analizaron con el índice de correlación de Spearman del paquete estadístico SPSS, versión 20 (IBM-SPSS, 2011).

\section{Resultados y Discusión}

Se colectaron un total de 146 cangrejos ermitaños, 68 (46,6 \%) en Punta Pajarito y $78(53,4 \%)$ en playa El Retén. Los valores in situ muestran para Punta Pajarito una preferencia hacia conchas de las familias Naticidae $(92,65 \%)$, mientras que en playa El 
Retén las conchas de mayor preferencia pertenecen a la familia Naticidae $(65,38 \%)$ Turritellidae (19,24\%) y Muricidae (6,42\%) (Tabla 1).

Tabla 1.

Frecuencia de abundancia de familias de gasterópodos entre los cangrejos ermitaños muestreados en playa El Reten, Bahía de Parita y Punta Pajarito, Golfo de Montijo.

Playa El Retén

Punta Pajarito

\begin{tabular}{lcccc}
\hline Familia & Frecuencia & \% & Frecuencia & \% \\
Naticidae & 51 & 65,38 & 63 & 92,65 \\
Turritellidae & 15 & 19,24 & 0 & 0 \\
Muricidae & 5 & 6,42 & 5 & 7,35 \\
Neritidae & 2 & 2,56 & 0 & 0 \\
Cassidae & 2 & 2,56 & 0 & 0 \\
Facioladiradae & 1 & 1,28 & 0 & 0 \\
Nassariidae & 1 & 1,28 & 0 & 0 \\
Columbellidae & 1 & 1,28 & 0 & 0 \\
Total & $\mathbf{7 8}$ & $\mathbf{1 0 0}$ & $\mathbf{6 8}$ & $\mathbf{1 0 0}$ \\
\hline
\end{tabular}

Fuente: En base a los datos recopilados en los sitios de muestreos. Elaboración propia.

Los resultados evidencian una preferencia hacia la ocupación de conchas de la familia Naticidae, en ambas localidades, siendo su mayor preferencia en Punta Pajarito $(92,65 \%)$ que en Playa el Retén $(65,38 \%)$, probablemente por la abundancia de dichas conchas en el área. Estos resultados concuerdan con los encontrados por Carranza et al (2008), quien señala que la preferencia por el uso de conchas es más similar entre poblaciones cercanas.

En playa El Retén, se seleccionaron al azar 31 organismos no ovígeros, de los cuales tres individuos pertenecían a la familia Coenobitidae y 28 a la familia http://revistas.up.ac.pa/index.php/guacamaya 
Diogenidae. Por su dominancia, a los cangrejos ermitaños pertenecientes a la familia

Diogenidae se les tomaron distintos parámetros biométricos como peso del organismo, y peso y volumen interno de la concha que portaban (Tabla 2).

Tabla 2.

Datos biométricos de cangrejos ermitaños de la familia Diogenidae y sus conchas, recolectados en playa El Retén, Bahía de Parita (Herrera).

$\mathbf{N}^{\circ}$

$\begin{array}{ccc}\text { Familia } & \text { Peso } & \text { Peso concha } \\ \text { Concha } & \text { organismo }(\mathrm{g}) & (\mathrm{g})\end{array}$

Volumen Relación Pesointerno-concha Volumen de la (ml) concha $(\mathrm{g} / \mathrm{ml})$

\begin{tabular}{|c|c|c|c|c|c|}
\hline 1. & Naticidadae & 5,10 & 25,60 & 7,00 & 3,66 \\
\hline 2. & Naticidadae & 1,30 & 4,80 & 3,00 & 1,60 \\
\hline 3. & Naticidadae & 5,50 & 15,20 & 8,00 & 1,90 \\
\hline 4. & Naticidadae & 2,30 & 21,70 & 5,10 & 4,25 \\
\hline 5. & Naticidadae & 2,40 & 31,60 & 6,50 & 4,86 \\
\hline 6. & Naticidadae & 1,20 & 14,10 & 3,20 & 4,41 \\
\hline 7. & Naticidadae & 1,50 & 16,90 & 5,00 & 3,38 \\
\hline 8. & Naticidadae & 2,30 & 16,50 & 4,50 & 3,67 \\
\hline 9. & Naticidadae & 1,40 & 25,30 & 5,00 & 5,06 \\
\hline 10. & Naticidadae & 1,30 & 12,60 & 4,50 & 2,80 \\
\hline 11. & Naticidadae & 2,90 & 17,30 & 5,00 & 3,46 \\
\hline 12. & Naticidadae & 2,00 & 10,10 & 5,00 & 2,02 \\
\hline 13. & Naticidadae & 1,30 & 14,60 & 4,00 & 3,65 \\
\hline 14. & Naticidadae & 2,00 & 29,60 & 7,00 & 4,23 \\
\hline 15. & Naticidadae & 2,80 & 14,30 & 4,50 & 3,18 \\
\hline 16. & Naticidadae & 0,80 & 4,10 & 2,50 & 1,64 \\
\hline 17. & Naticidadae & 0,50 & 4,10 & 1,50 & 2,73 \\
\hline
\end{tabular}


cuAcAmaYa

18. Naticidadae

19. Naticidadae

20. Muricidae

21. Muricidae

22. Muricidae

23. Muricidae

24. Muricidae

0,50

2,80

2,00

25. Columbellidae

26. Neritidae

27. Cassidae

28. Cassidae

\section{Fuente: En base a los datos recopilados en playa El Retén. Elaboración propia}

Al correlacionarse las variables, peso del organismo y peso de la concha, el coeficiente de correlación de Spearman indica que ambas variables se correlacionan positivamente a un nivel significativo $(\mathrm{p}=0,006)$, concordando con lo argumentado por diversos autores, en referencia a que el peso de las conchas es la variable que mejor describe la asociación entre los cangrejos ermitaño y sus conchas (Wilber, 1990; Mantelatto \& Domiciano, 2002; Carranza et al., 2008; Sardar et al., 2019).

No obstante, al encontrarse una correlación significativa entre el peso de la concha y su volumen interno $(\mathrm{p}=0,000)$ y no obtenerse correlación significativa entre el peso del organismo y la relación peso-volumen de la concha $(\mathrm{p}=0,386)$, se asume que en la playa El Retén, la elección de conchas por cangrejos ermitaños obedece a su disponibilidad. Esto queda evidenciado por el gran número de conchas vacías encontradas en el área, y por investigaciones como la realizada por Ribeiro et al. (2018), donde manifiesta que la especie Pagarus brevidactylus tiene una patrón de ocupación de conchas de acuerdo a la localidad, por ende a la disponibilidad de conchas.

\section{Conclusiones}

El presente estudio constituye un primer esfuerzo en el área para determinar los patrones ecológicos que interactúan en la selección de conchas por cangrejos ermitaños. Lejos de ser exhaustivas, las conclusiones aquí presentadas deben motivar futuras 
investigadores que ahonden en los criterios utilizados por cangrejos ermitaños en la selección de conchas. Entre tanto, presentamos las siguientes conclusiones:

En Punta Pajarito, Golfo de Montijo (Veraguas) y playa El Retén, Bahía de Parita (Herrera), las conchas de mayor preferencia por cangrejos ermitaños corresponden a las de familia Naticidae.

Para los organismos colectados en playa El Retén, la no correlación entre el peso de los cangrejos ermitaños y la relación peso/volumen de su concha, aunado al alto número de conchas vacías encontradas en el área, indican que la disponibilidad de conchas es el criterio que mejor puede explicar la selección de conchas en dicho sitio por los cangrejos ermitaños.

\section{Referencias Bibliográficas}

Angel, J.E. (2000). Effects of shell fit on the biology of the hermit crab Pagurus longicarpus (Say). Journal of Experimental Marine Biology and Ecology 243: 169-184. Recuperado de:

https://www.sciencedirect.com/science/article/pii/S0022098199001197?via \%3Dihub

Arguelles-Tico, A., Álvarez, F., Alcaraz G. (2010). Shell utilization by the hermit crab Clibanarius antillensis Stimpson 1862 (Crustacea Anomura) in intertidal rocky pools at Montepio, Veracruz, Mexico. Tropical Zoology 23: 63-73. Recuperado de: https://n9.cl/znq3

Arrasate-López, M. (2008). Comportamiento del cangrejo ermitañom Clibanarius aequabilis (Decapada: Anomura) en la selección de la concha. Anales Universitarios de Etología 2:114-118. Recuperado de:https://accedacris.ulpgc.es/bitstream/10553/513/1/4645.pdf

Bertness, M.D. (1980). Shell preference and utilization patterns in littoral hermit crabs of the Bay of Panama. Journal of Experimental Marine Biology and Ecology 48: 1-16. Recuperado de: https://www.sciencedirect.com/science/article/pii/0022098180900027 
Biagi, R., Meireles, A.L., Scelzo, M.A. y Mantellato, F.L. (2006). Estudio comparativo sobre elección de conchas en el cangrejo ermitaño endémico del Atlántico sudoccidental Loxopagurus loxochelis de Brasil y Argentina. Revista Chilena de Historia Natural 79: 481-487. Recuperado de: https://scielo.conicyt.c1/scielo.php?pid=S0716078X2006000400007\&script=sci_a $\underline{\text { rttext }}$

Carranza, A., Segura, A., López, J. y Rubio, L. (2008). Shell use patterns of the hermit crab Loxopagurus loxochelis (Decapoda: Diogenidae) in Cerro Verde-La Coronilla (Rocha-Uruguay). Comunicaciones de la Sociedad Malacológica del Uruguay 9 (91): 139 - 145. Recuperado de: https://www.redalyc.org/pdf/524/52412049002.pdf

Cipresso P, P. H., Zancaner J., J. y Jacobucci, G. B. (2009). Ocupação de conchas e utilização de microambientes por caranguejos ermitões (Decapoda, Anomura) na Praia da Fortaleza, Ubatuba, São Paulo. Biotemas, 22 (2): 65-75.

DOI: https://doi.org/10.5007/2175-7925.2009v22n2p65

Conover, M. (1978). The importance of various shell characteristics to the shellselection behavior of the hermit crabs. Journal of Experimental Marine Biology and Ecology 32: 131-142. Recuperado de: https://www.sciencedirect.com/science/article/pii/0022098178901119

Dahlet. L. L., Checon, H. H., Yokoyama. L.Q. y Turra, A. (2019): Patterns of shell utilization by hermit crabs as a proxy of gastropod population_dynamics. Marine Biology Research.Recuperado de:https://doi.org/10.1080/17451000.2019.1662446

Fisher, W., Krupp, F., Schneider, W., Sommer, C., Carpenter, K. E, y Niem, V. H. (1995). Guía para la identificación de especies para los fines de la pesca Pacífico Centro-Central. FAO.1813 pp.Recuperado de: https://ci.nii.ac.jp/naid/10025862236/

Guillén, F. C. y Osorno, J. L. (1993). Elección de concha en Coenobita compressus (Decapoda: Coenobitidae). Rev. Biol. Trop 41 (1): 65-72.Recuperado de: https://revistas.ucr.ac.cr/index.php/rbt/article/view/23305

Hazlett, A. (1996). Assessments during shell exchanges by the hermit crab Clibanarus vittatus: the complete negotiator. Animal Behaviour, 51:567- 573.Recuperado de: https://www.sciencedirect.com/science/article/abs/pii/S0003347296900603 
GUACAMAYA

IBM-SPSS (2011). “Índice de correlación de Spearman”. IBM SPSS Statistics v. 20.0. Programa Estadístico para Ciencias Sociales. v. 20.0. Statistical Program for Social Sciences (SPSS). United States of American: Guide to IBM SPSS: For Versión 20.0

Jeremy, Y. y Patria, M. P. (2020). Strawberry Hermit Crab (Coenobita perlatus, H. Milne Edwards, 1837) Gastropod Shell Utilization Pattern According to the Type and Size. AIP Conference Proceedings 2242, 050021. Recuperado de: $\underline{\text { https://doi.org/10.1063/5.0008042 }}$

Keen, A. M. (1971). Sea shells of tropical West America. Second edition. Stanford University. Press. 1064 pp.Recuperado de: https://agris.fao.org/agris$\underline{\text { search/search.do? recordID }=\text { US201300477796 }}$

Mantelatto, F. L. M. y Domiciano, L.C.C. (2002). Pattern of shell utilization by the hermit crab Paguristes tortugae (Diogenidae) from Anchieta Island, southern Brazil. Scientia Marina 66: 265-272.Recuperado de: http://scientiamarina.revistas.csic.es/index.php/scientiamarina/article/view/585

Mantelatto, F. L., Biagi, R., Meireles, A. L. y Scelzo, M. A . (2007). Shell preference of the hermit crab Pagurus exilis (Anomura: Paguridae)_from Brazil and Argentina: a comparative study. Rev. Biol. Trop. 55: 153-162. Recuperado de: https://www.redalyc.org/pdf/449/44909919.pdf

Martínez C, B., Campos, N.H. y Bermúdez, A. (2012). Distribución de cangrejos ermitaños (Anomura: Paguroidea) en el mar Caribe colombiano. Rev. Biol. Trop. 60 (1): 233-252. Recuperado de: https://n9.cl/d1tc

Monteforte, M. y Leija- Tristán, A. (1990). Asociaciones entre cangrejos ermitaños y conchas de moluscos gastrópodos en el Pacífico de Sudcalifornia. Rev. BioI. Trop. 38 (2A): 283-288. Recuperado de: https://revistas.ucr.ac.cr/index.php/rbt/article/view/25373

Murillo, A., Burbano, C. y Olano, J. (2003). Cangrejo ermitaño busca casa móvil. Hipótesis: 23- 29. Recuperado de: https://dialnet.unirioja.es/servlet/articulo?codigo $=7148980$

Osorno, J. L., Fernández-Casillas, L., y Rodríguez-Juárez, C. (1998). ¿Los cangrejos ermitaños buscan conchas grandes y livianas?: evidencia de intercambios de 
conchas naturales e inducidos por el campo. Journal of Experimental Marine Biology and Ecology, 222 (1-2): 163-173. Recuperado de: https://www.sciencedirect.com/science/article/pii/S002209819700155X

Pogio, N.A. (2007). Parámetros de selección de conchas por parte del cangrejo ermitaño Clibanarius aequabilis (Crustacea, Decapoda). Anales Universitarios de Etología 1:1-6. Recuperado de:https://accedacris.ulpgc.es/bitstream/10553/1497/1/4311.pdf

Rendón-Salinas, E. (2000). Elección de conchas, por parte de cangrejos ermitaños Coenobita compressus, de dos géneros diferentes de gasterópodos. Ciencia y Mar 4: 51-54. Recuperado de: https://n9.cl/v3m11

Ribeiro S, A., Galli, G. M., Stanski, G., De Biasi, J. B., Davanso T. M., Cobo, V.J. y Castilho, A. L. (2019) Shell occupation as a limiting factor for Pagurus brevidactylus (Stimpson, 1859) in the Marine_State Park of Laje de Santos, Brazil. Invertebrate Reproduction \& Development 63 (1): 1-10. Recuperado de: https://doi.org/10.1080/07924259.2018.1513087

Romero, A. (1973). El curioso cangrejo ermitaño. Algo. Revista Quincenal de divulgación científica, técnica y cultural 220: 141-144. Recuperado de:https://spark.siue.edu/bio_fac/9/

Sardar, S., Ghosh, D., Kumar G., P., Bhattacharjee, K. y Pal, K. (2019). First report on the use of gastropod shells by hermit crabs from the eastern coast, India. The Pharma Innovation Journal 8(3): 22-30. Recuperado de: http://www.thepharmajournal.com/archives/2019/vol8issue3/PartA/8-2-89136.pdf

Wilber Jr, T. P. (1990). Influence of size, species and damage on shell selection by the hermit crab Pagurus longicarpus. Marine Biology 104: 31-39.Recuperado de: https://link.springer.com/article/10.1007/BF01313154 